\title{
L'insaisissable notion de surveillance en droit pénal
}

Philippe Collet, Maître de conférences à l'université de Rennes 1, IODE (UMR CNRS 6262)

***

L'essentiel

Omniprésente en droit pénal substantiel et formel, la surveillance apparait comme une notion fuyante. Conforme à la réalité juridique, celle-ci ne fait l'objet d'aucune définition légale ou jurisprudentielle et les travaux de la doctrine sont exclusivement consacrés soit aux surveillances du droit pénal, soit à celles de la procédure pénale. Ainsi, cette notion n'est jamais étudiée dans sa globalité. Une telle lacune s'explique par la nature duale de la surveillance, à laquelle correspond une double fonction. L'unité de la notion reste aussi introuvable en raison de l'absence d'un ou de plusieurs critères idoines.

Surveillance, which is omnipresent in substantive and formal criminal law, appears to be an elusive concept. In accordance with legal reality, there is $n^{\circ}$ legal or jurisprudential definition of the latter and the work of the doctrine is exclusively devoted to either criminal law or criminal procedure supervision. Thus, this notion is never studied in its entirety. This is due to the dual nature of surveillance, which has a dual function. The unity of the concept also remains impossible to find due to the absence of one or more suitable criteria

Parce qu'elle est omniprésente, la surveillance soulève de manière récurrente des questions essentielles au regard des libertés individuelles 眘(1). Elle s'avère nécessaire au recueil d'informations et de renseignements, mais aussi à l'activité de contrôle des personnes. On la discerne, par exemple, lorsqu'un employeur recourt à des mesures de surveillance de la correspondance et des autres communications de l'un de ses employés du 24 janvier 2015 relative au renseignement苜(3). Celui-ci prévoit la sonorisation, la surveillance des communications électroniques internationales, le placement sous surveillance électronique mobile, etc. 眘(4)

Cest néanmoins en droit pénal, substantiel comme formel, que la surveillance trouve son domaine d'élection. En droit pénal de fond, elle apparaît au cours de l'exécution des peines afin d'éviter l'incarcération tout en astreignant le délinquant à un contrôle indispensable. Les mesures concernées sont la surveillance judiciaire, la surveillance de sûreté ou le placement sous surveillance électronique自(5). De son côté, la procédure pénale permet aux enquêteurs et aux magistrats d'accomplir différents actes qui consistent à surveiller des individus. Il existe une surveillance des personnes ou de l'acheminement ou du transport des objets, biens ou produits tirés de la commission d'infractions ou servant à les commettre眘(6). Mais les officiers de police judiciaire peuvent effectuer de nombreuses mesures dont la fonction est, en fin de compte, similaire : infiltration, écoutes téléphoniques, sonorisation et fixation d'images, géolocalisation, IMSI catcher 眘(7)... La surveillance, « qui peut certes représenter un danger pour la vie privée, correspond à une exigence de sécurité et de maintien de l'ordre public autant qu'elle représente un moyen efficace d'investigation »眘(8). Lors de la recherche de preuves par les parties privées, elle peut être exploitée par la victime de l'infraction, s'appuyant par exemple sur la vidéoprotection眘(9), ou par la personne poursuivie, voire par un enquêteur privé, dénommé agent de recherches privées par la loi苜(10). La multiplication des acteurs privés apportant des preuves au procès pénal ne saurait pour autant occulter le rôle primordial des autorités publiques en matière de surveillance 眘(11). Quoi qu'il en soit, cette notion mérite une attention particulière en essayant d'identifier son ou ses critères.

Eu égard à son existence au sein de plusieurs normes, notamment dans les dispositions des Codes pénal et de procédure pénale ainsi qu'en jurisprudence曾 (12), la surveillance appartient à la réalité juridique苜(13). Une étude peut alors être menée à son sujet en l'absence de toute définition légale ou jurisprudentielle曾(14). Au sens courant, elle se définit comme «l'action de surveiller, de contrôler quelque chose, quelqu'un » ou comme « le contrôle suivi auquel on est soumis ; observation »眘(15). Le vocabulaire juridique CORNU évoque, quant à lui, « l'action de veiller sur une personne ou une chose dans l'intérêt de celle-ci, ou de surveiller une personne ou une opération pour la sauvegarde d'autres intérêts -, action préventive qui, fondée sur la vigilance de celui qui surveille (marquée par des actes de vérification et de contrôle), s'applique à l'action d'autrui dans le temps (au développement, au devenir de ce qui est surveillé) »眘(16).

Ces quelques éléments fournissent une première approche de la surveillance. Toutefois, ils demeurent insuffisants pour l'appréhender complètement. Les travaux de la doctrine, qui lui sont consacrés, ne se révèlent guère plus éclairants. Ils s'attachent surtout à examiner des surveillances en particulier, soit celles du droit pénal, soit celles de la procédure pénale. Cest dire qu'aucune étude ne semble s'intéresser à ce concept dans son ensemble, à celui-ci pris dans sa globalité.

Une telle lacune, a priori étonnante, est en réalité compréhensible. Notion transversale du droit pénal lato sensu, la surveillance s'avère très difficile à cerner. Elle apparaît fuyante et, par conséquent, rebelle à toute définition. Au fond, son caractère insaisissable procède de sa différence de nature (I), à laquelle s'ajoute une divergence de ses critères (II).

\section{I - La différence de nature}

Procéder à un examen sommaire de la surveillance conduit aussitôt à souligner son manque d'unité. La notion présente en effet une dualité manifeste en droit pénal. Elle possède une nature hétérogène, s'analysant tantôt comme un acte d'instruction aux fins de preuve de l'infraction (A), tantôt comme un acte de juridiction aux fins de prévention de la récidive (B).

\section{A - Un acte d'instruction aux fins de preuve de l'infraction}


En théorie, la surveillance exercée en matière pénale se distingue bien de la surveillance administrative. Il est acquis que « les finalités de l'activité de renseignement sont fondées sur la dangerosité potentielle d'une situation, non sur l'existence de soupçons déterminés à l'égard d'un individu, contrairement aux solutions prévalant en procédure pénale qui requièrent, dans le cadre d'une mission de police judiciaire, que les actes d'enquête soient fondés sur des raisons plausibles de soupçonner la commission d'une infraction »首(17). Mais de façon concrète, la distinction reste complexe entre la police administrative destinée à prévenir les infractions et la police judiciaire chargée de constater celles-ci曾 (18). Il faut pourtant s'efforcer de l'établir, car « les mesures de police administrative échappent aux garanties imposées à une procédure judiciaire et, pour cette raison, ne peuvent être utilisées comme preuve de la commission d'une infraction »眘(19).

Si le Conseil constitutionnel tient à rappeler ce partage de compétence首(20), celui-ci montre malgré tout une certaine porosité. « Des chevauchements existent, d'une part parce que la même situation peut être comprise comme la prévention d'une infraction matérielle et comme la consommation d'une infraction-obstacle et d'autre part parce que des opérations de renseignement, menées dans un but préventif, pourraient ensuite être portées à la connaissance du ministère public en vue d'éventuelles poursuites pénales »菌(21). En outre, les services de renseignement disposent de mesures identiques à celles confiées à la police judiciaire : interceptions, infiltrations, sonorisations, etc. ; et si les informations récoltées « ne peuvent pas directement servir de preuve pour une infraction commise, elles permettront (et permettent déjà) de faire démarrer une enquête ou une instruction »眘(22). En dépit de la grande proximité des actes de police administrative avec ceux réalisés au cours d'une enquête de police judiciaire, seuls ces derniers doivent retenir l'attention car l'étude n'a pas pour objet les techniques probatoires utilisées dans le cadre d'enquêtes proactives

Ainsi, les enquêteurs et les magistrats mènent des investigations dont l'objet est de surveiller les personnes et les biens en procédure pénale. Intercepter les correspondances émises par la voie des télécommunications, sonoriser et fixer les images de certains lieux, capter des données informatiques, infiltrer un réseau criminel, géolocaliser une personne, un véhicule ou un téléphone, etc., constituent autant de surveillances permettant de recueillir des informations nécessaires à l'enquête. Du point de vue de leur nature, ces actes demeurent dissemblables des mesures d'administration judiciaire ou bien des décisions juridictionnelles 首(24). Insusceptibles de se rattacher à ces deux catégories, ils sont des actes de police judiciaire ou d'instruction selon le cadre juridique de la procédure suivie.

À l'origine, l'acte d'instruction a été analysé comme « l'acte qui tend à la recherche et à la constitution d'une preuve, et qui est accompli, après l'ouverture du procès pénal, soit par un juge d'instruction ou sur délégation de ce dernier, par un officier de police judiciaire, soit par un autre juge. L'acte d'instruction, c'est essentiellement un acte de justice »首(25). Puis, il a été défini comme un acte qui tend à la manifestation de la vérité, réalisé par un juge possédant au moins une aptitude à un pouvoir juridictionnel眘(26). Ce serait cette qualité de l'auteur de l'acte, élément subjectif, qui serait déterminante et primerait le critère du moment auquel l'acte de recherche intervient菌(27). Finalement, un acte d'instruction «n'est qu'un acte de poursuite assorti de garanties »首(28).

Mais à vrai dire, « les actes d'investigation exécutés par le juge d'instruction ne sont pas, en nature, différents des actes de police judiciaire, puisqu'il s'agit, dans les deux cas, d'actes d'administration de la preuve. Comme les actes de police judiciaire, ces actes d'instruction relatifs aux investigations ont pour objet la recherche coercitive des preuves et pour finalité la manifestation de la vérité »菌 (29). Dotés de la même fonction, ces deux actes ont le même effet interruptif de prescription de l'action publique苜(30). Qu'elles interviennent au cours d'une enquête, préliminaire ou de flagrance, ou même lors d'une information, les mesures de surveillance méritent donc d'être qualifiées d'actes d'instruction.

De telles mesures présentent forcément des différences entre elles. Certaines sont visuelles, comme la surveillance stricto sensu ou l'infiltration; d'autres, envisagées comme « une forme de guet », consistent en des enregistrements effectués avec des dispositifs techniques ou supposent l'emploi de moyens technologiques clandestins 首(31). Toutes sont en revanche secrètes afin de conserver leur efficacité, l'objectif étant de permettre la recherche des auteurs et la réunion des preuves de l'infraction. La dissimulation a été admise en droit européen s'agissant, par exemple, de l'intervention d'agents infiltrés, mais une limite a été posée. En effet, «si elle peut agir en secret, la police ne peut pas provoquer la commission d'une infraction »眘(32). Cette règle apparaît logique, les activités de surveillance entraînant une ingérence de l'État au sens de l'article 8 de la Convention de sauvegarde des droits de l'homme眘(33). Elle s'applique à tous les actes de surveillance accomplis en procédure pénale眘(34).

Ceux-ci, dont certains ont été instaurés par la loi du 3 juin 2016, se révèlent très attentatoires à la vie privée des personnes 苜(35). « Si le progrès dans les nouvelles méthodes d'enquête - concomitant à la modernisation de la criminalité - est commandé par le souci d'accrôtre la réponse pénale et l'efficacité de la répression, ces technologies sont des moyens qui s'immiscent toujours davantage dans la sphère d'intimité de l'individu 》眘(36). Une différence de degré de l'intrusion dans la vie privée existe alors selon les modes de preuve utilisés. Ainsi, pour la Cour de Strasbourg, les opérations de géolocalisation doivent être considérées comme étant moins attentatoires à la vie privée d'une personne que les écoutes téléphoniques, par exemple眘(37). Il faut reconnaître que les nouvelles techniques numériques d'enquête, tel l'IMSI catcher ou la captation des données informatiques, sont particulièrement intrusives 眘(38). Elles se généralisent d'ailleurs de façon progressive, soit par leur introduction en droit commun, soit par l'extension constante du champ d'application des procédures dérogatoires 眘(39). Conscient de cette évolution, le législateur vient d'introduire un alinéa à l'article préliminaire du Code de procédure pénale dont l'objectif est de soumettre davantage les investigations constitutives de surveillances à la garantie judiciaire. Désormais, « au cours de la procédure pénale, les mesures portant atteinte à la vie privée d'une personne ne peuvent être prises, sur décision ou sous le contrôle effectif de l'autorité judiciaire, que si elles sont, au regard des circonstances de l'espèce, nécessaires à la manifestation de la vérité et proportionnées à la gravité de l'infraction »菌(40).

Ces actes de la procédure forment, en définitive, des actes d'instruction ayant une finalité probatoire et pouvant faire l'objet d'une requête en annulation眘(41). Les décisions qui les autorisent doivent être motivées 眘(42); mais le législateur prend soin de préciser, en général, qu'elles sont dépourvues de caractère juridictionnel et insusceptibles de recours rencontre en droit pénal. De nature distincte, elle vient expliquer la dualité de cette notion.

\section{B - Un acte de juridiction aux fins de prévention de la récidive}


En plus de la surveillance occulte destinée à obtenir des informations et à établir la preuve de l'infraction, le législateur organise une « surveillance à distance » comme substitut à l'incarcération et « manifeste »首(44). Lors d'une information judiciaire, le juge d'instruction ou le juge des libertés et de la détention considère parfois déraisonnable de laisser la personne mise en examen en liberté totale. Aussi peut-il décider, avec l'accord ou à la demande de celle-ci, de l'assigner à résidence avec surveillance électronique si elle encourt au minimum une peine de deux ans d'emprisonnement correctionnel苜(45). Dans une telle situation, la surveillance demeure apparente et de surcroît consentie. À l'instar d'un placement sous contrôle judiciaire ou en détention provisoire, l'assignation à résidence avec surveillance électronique ne saurait être perçue comme un acte d'instruction. Elle constitue plutôt une décision de justice. De multiples actes de surveillance revêtant cette nature, il convient de définir l'acte juridictionnel, du moins d'évoquer brièvement la façon dont il a été appréhendé.

À cet égard, « le critère de l'acte juridictionnel, par opposition à celui de l'acte administratif, n'a jamais cessé d'aiguiser la curiosité de la doctrine, à défaut de fixer l'attention du législateur qui ne s'en préoccupe guère »眘(46). En l'absence de toute définition légale, les auteurs ont alors avancé différents critères, formels et matériels, en vue de le cerner囬(47). Une combinaison de certains de ces critères a été progressivement admise. Parmi eux, celui de l'existence d'une contestation (ou d'un litige) à trancher domine l'analyse苜(48). La contestation apparaît comme un « incident de l'ordonnancement juridique », qui se caractérise par l'autorité de celui qui y procède et la conformation au droit, c'est-à-dire le moyen de réaliser la fonction, d'apaiser le conflit眘(49).

De son côté, l'article 6 de la Convention de sauvegarde des droits de l'homme évoque la fonction de juger, qui suppose celle de trancher des contestations 首 (50). Le juge règle le litige par une décision obligatoire, celle-ci devant être prise conformément au droit 眘 (51). Selon la Cour de Strasbourg, juger consiste à trancher, sur la base de normes de droit et à l'issue d'une procédure organisée, toute question relevant de sa compétence首(52). La fonction juridictionnelle se résume donc à trancher les litiges en disant le droit目(53). La jurisdictio signifie le pouvoir qui appartient au juge, saisi d'une contestation élevée sur un cas concret, d'y mettre fin en constatant le droit applicable à la situation litigieuse et en ordonnant les mesures propres à en assurer le respect眘(54). Il faut bien sûr que le juge soit un tiers par rapport au litige, car l'acte juridictionnel tranche entre deux prétentions et met un terme au conflit en conférant à l'une d'elles force de vérité légale眘 (55).

Ces quelques éléments contribuent à mieux saisir l'acte juridictionnel. Soulignant la spécificité essentielle du droit pénal au regard du procès civil, la doctrine pénaliste constate la place respective du juge et de la loi dans l'existence même de celui-là. Le droit pénal n'existe pas sans loi, et surtout, « sans juge, il n'y a pas de droit pénal du tout : ce sont en effet uniquement des juges, qui mettent en oeuvre le droit pénal, en prenant des décisions privatives ou restrictives de liberté au stade de l'instruction, en reconnaissant ou non une culpabilité et, le cas échéant, en prononçant et/ou appliquant des peines ou des mesures de sûretés. Or, là où il y a des juges, il y a de fortes chances que l'on trouve la trace de la jurisdictio »眘(56). Cette singularité expliquerait le désintérêt des auteurs de procédure pénale pour le concept d'acte juridictionnel眘(57). Une théorie sur cet acte mériterait pourtant d'être formulée en raison du « développement incontrôlé, sinon anarchique, des mesures d'administration judiciaire »眘(58). Opposée à l'acte juridictionnel, la notion de mesure d'administration judiciaire a néanmoins été évincée dans la phase de l'exécution des peines, à titre principal par les lois du 15 juin 2000 et du 9 mars 2004眘(59).

Cest précisément lors de la condamnation pénale et de l'exécution des peines que de nombreuses surveillances, fondées sur des décisions juridictionnelles, sont instituées en droit français contemporain. Au moment de la condamnation, la juridiction de jugement qui prononce un emprisonnement peut ordonner qu'il soit sursis à son exécution, le condamné étant placé sous le régime de la mise à l'épreuve眘 (60). Comme le sursis simple, le sursis avec mise à l'épreuve suspend l'exécution de la peine d'emprisonnement prononcée眘(61). Mais il permet d'astreindre le condamné à des mesures de contrôle énoncées à l'article 132-44 du Code pénal et à lui imposer des obligations particulières prévues à l'article 132-45 dudit Code. Ces mesures et obligations diverses tendent à surveiller le délinquant et, au-delà, à lutter contre la récidive眘 (62). Il ne fait aucun doute que la décision prononçant un tel sursis possède une nature juridictionnelle.

Une autre façon de surveiller le condamné requiert d'utiliser un dispositif technologique, autrement dit de le placer sous surveillance électronique. Aux termes de l'article 132-26-1 du Code pénal, la juridiction de jugement peut, lorsqu'elle prononce une peine d'emprisonnement de deux ans au plus, ou pour une personne en état de récidive légale d'un an au plus, décider que la peine sera exécutée en tout ou partie sous le régime du placement sous surveillance électronique à l'égard du condamné qui justifie de l'exercice d'une activité professionnelle, même temporaire, de la recherche d'un emploi, de sa participation essentielle à la vie de sa famille, de la nécessité de suivre un traitement médical, etc. La décision de placement sous surveillance électronique fixe ne s'analyse ni en une mesure d'administration judiciaire ni en un acte d'information. Elle demeure un acte juridictionnel, qui ne peut être pris qu'avec l'accord du prévenu préalablement informé qu'il peut demander à être assisté par son avocat, le cas échéant désigné d'office par le bâtonnier à sa demande, avant de donner son accord眘 (63). La décision du juge de l'application des peines, adoptée en vertu de l'article 723-7 du Code de procédure pénale眘(64), présente le même caractère. Ce juge peut prévoir que la peine s'exécutera sous le régime du placement sous surveillance électronique soit en cas de condamnation à une ou plusieurs peines privatives de liberté dont la durée totale n'excède pas deux ans, soit lorsqu'il reste à subir par le condamné une ou plusieurs peines privatives de liberté dont la durée totale n'excède pas deuxans 眘(65).

Dans l'arsenal répressif, les magistrats disposent encore du placement sous surveillance électronique mobile, instauré par la loi $\mathrm{n}^{\circ} 2005-$ 1549 du 12 décembre 2005 relative au traitement de la récidive des infractions pénales. Cette mesure, qui participe du suivi socio-judiciaire 眘(66), est une mesure de sûreté⿴囗⿱一一⿱⿴囗十丌(一)(67), ordonnée par le tribunal correctionnel ou la cour d'assises à l'encontre d'une personne majeure condamnée à une peine privative de liberté d'une durée égale ou supérieure à sept ans (cinq ans dans certains cas), et dont une expertise médicale a constaté la dangerosité, lorsque cette mesure s'avère indispensable pour prévenir la récidive à compter du jour où la privation de liberté prend fin苜 (68). En pratique, le condamné a l'obligation de porter pour une durée de deux ans, renouvelable une fois en matière délictuelle et deux fois en matière criminelle, un émetteur permettant à tout moment de déterminer à distance sa localisation sur l'ensemble du territoire national眘 (69). Si le placement ne peut s'appliquer sans le consentement de l'intéressé, un refus de sa part lui fait encourir une condamnation pénale surveillance électronique susmentionné dispositif mobile, quant à lui, intervient après l'accomplissement de la peine, afin de prévenir tout risque de récidive, ce que la loi traduit explicitement en le qualifiant de "mesure de sûreté" »眘(72). 
entre la dangerosité et ce droit眘(74), résulte une prolifération des mesures de neutralisation des individus supposés dangereux苜(75). Toutes ces mesures de sûreté, dont certaines se traduisent par la mise en oeuvre de surveillances étroites, visent à éviter la récidive. Ainsi les articles 723-29 et suivants du Code de procédure pénale prévoient-ils la surveillance judiciaire, créée par la loi du 12 décembre 2005 眘 (76). Pour la résumer囬 (77), le tribunal de l'application des peines peut ordonner, sur réquisitions du parquet, à titre de mesure de sûreté et aux seules fins de prévenir une récidive dont le risque paraît avéré, le placement sous surveillance judiciaire des personnes dangereuses condamnées à une peine privative de liberté d'une durée au moins égale à sept ans pour un crime ou un délit pour lequel le suivi sociojudiciaire est encouru, ou d'une durée d'au moins cinq ans pour un crime ou un délit commis à nouveau en récidive légale. Débutant dès que le condamné a fini l'exécution de sa peine privative de liberté, la surveillance ne peut excéder une durée correspondant au crédit de réduction de peine et aux réductions de peines supplémentaires dont il a profité et qui n'ont pas fait l'objet d'une décision de retrait㘢(78). Elle soumet, par exemple, l'intéressé au respect des obligations prévues au titre de la mise à l'épreuve ou à la surveillance électronique mobile en cas de possibilité technique首(79). Le placement sous surveillance judiciaire doit être décidé avant la date de la libération du condamné par un jugement rendu conformément aux dispositions de l'article 712-6 du Code de procédure pénale眘(80). Lors du débat contradictoire, lintéressé est obligatoirement assisté par un avocat choisi par lui ou désigné, à sa demande, par le bâtonnier. Et le jugement précise les obligations auxquelles il est tenu, ainsi que la durée de celles-ci苜(81).

Instituée par la loi n ${ }^{\circ}$ 2008-174 du 25 février 2008, la surveillance de sûreté comporte en outre des obligations identiques à celles aménagées pour la surveillance judiciaire, notamment linjonction de soins et le placement sous surveillance électronique mobile眘(82). Elle ne peut être prononcée qu'après expertise médicale constatant la persistance de la dangerosité et dans le cas où les obligations résultant de l'inscription dans le fichier judiciaire national automatisé des auteurs d'infractions sexuelles ou violentes s'avèrent insuffisantes pour prévenir la récidive眘(83). Elle peut aussi être adoptée si la rétention de sûreté n'est pas prolongée ou s'il y est mis fin alors que la personne présente toujours des risques de récidive首(84). Mais si le condamné se soustrait à ses obligations, son comportement révélant une probabilité très élevée d'accomplir à nouveau l'une des infractions énoncées à l'article 706-53-13 du Code de procédure pénale, il peut être placé en urgence dans un centre socio-médico-judiciaire de sûreté à condition toutefois qu'un renforcement des obligations de la surveillance de sûreté apparaisse insuffisant pour prévenir la récidive眘(85). En ce qui concerne sa nature juridique, la surveillance de sûreté - comme la rétention de sûreté - ne constitue «ni une peine ni une sanction ayant le caractère d'une punition », elle n'est pas une " mesure répressive »眘(86). Mesure très restrictive de liberté, la décision qui l'ordonne répond tout à fait aux critères de l'acte juridictionnel. Le placement sous surveillance de sûreté peut d'ailleurs faire l'objet des recours prévus à l'article 706-53-15眘(87).

Finalement, ces différentes mesures sont des décisions juridictionnelles et témoignent, tout au moins pour les plus attentatoires aux libertés individuelles, d'une volonté législative de mettre la dangerosité sous surveillance首(88). Leur objectif apparaît préventif : il s'agit d'éviter la commission d'une nouvelle infraction. Dès lors, elles se distinguent nettement des surveillances constitutives d'actes d'instruction, accomplies aux fins de preuve de l'infraction. En droit pénal, la surveillance possède donc une double nature à laquelle correspond une fonction propre. Cette dualité de la notion explique, en grande partie, les difficultés à l'appréhender de manière globale. À ce premier obstacle, s'en ajoute un second qui tient à la complexité de fournir à celle-ci des critères adéquats.

\section{II - La divergence des critères}

Compte tenu de son hétérogénéité, la surveillance peine à disposer d'une certaine unité en droit pénal. Celle-ci est encore contrariée par l'impossibilité de trouver un critère satisfaisant à cette notion transversale. Bien entendu, plusieurs critères peuvent être identifiés afin de la saisir ; mais aucun ne semble adéquat à la définir. En effet, tant les critères personnel et matériel (A), que temporel et formel (B), révèlent leur insuffisance.

\section{A - L'insuffisance des critères personnel et matériel}

Dans les études consacrées aux notions juridiques, le premier critère examiné est, en principe, le critère organique ou personnel. Pareille démarche semble logique compte tenu de sa simplicité et du fait que celui-ci se conçoit aisément. Ce critère peut tout à fait s'appliquer à la surveillance en droit pénal. Il se divise alors, pour comprendre un aspect actif et un autre passif.

D'un point de vue actif, il conduit à s'intéresser à l'auteur de la surveillance. Celui qui exerce cet acte constitue généralement une autorité publique, un enquêteur la plupart du temps 眘(89). Ainsi, les surveillances policières s'avèrent fréquentes en procédure pénale. Aux termes de l'article 706-80, alinéa 1, du Code de procédure pénale, « les officiers de police judiciaire et, sous leur autorité, les agents de police judiciaire, après en avoir informé le procureur de la République et sauf opposition de ce magistrat, peuvent étendre à l'ensemble du territoire national la surveillance de personnes contre lesquelles il existe une ou plusieurs raisons plausibles de les soupçonner d'avoir commis l'un des crimes et délits entrant dans le champ d'application des articles 706-73, 706-73-1 ou 706-74 ou la surveillance de l'acheminement ou du transport des objets, biens ou produits tirés de la commission de ces infractions ou servant à les commettre »眘(90). De même, l'opération d'infiltration, qui consiste « à surveiller des personnes suspectées de commettre un crime ou un délit en se faisant passer, auprès de ces personnes, comme un de leurs coauteurs, complices ou receleurs ", est effectuée par " un officier ou un agent de police judiciaire spécialement habilité dans des conditions fixées par décret et agissant sous la responsabilité d'un officier de police judiciaire chargé de coordonner l'opération »眘(91). La géolocalisation苜(92), ou les « autres techniques spéciales d'enquête »苜(93) telles les sonorisations et fixations d'images de certains lieux ou véhicules 眘(94), illustrent encore le rôle prépondérant de la police judiciaire en matière de surveillances首(95).

Cette surveillance policière s'accompagne en plus d'une surveillance judiciaire dont la progression est constante depuis une quinzaine d'années. Autrement dit, l'auteur de la surveillance peut très bien être un magistrat. Le juge de l'application des peines a, par exemple, la faculté de prévoir que la peine s'exécutera sous le régime du placement sous surveillance électronique défini par l'article 132-26-1 du Code pénal soit en cas de condamnation à une ou plusieurs peines privatives de liberté dont la durée totale n'excède pas deux ans, soit lorsqu'il reste à subir par le condamné une ou plusieurs peines privatives de liberté dont la durée totale n'excède pas deux ans 眘(96). À titre de mesure de sûreté, les juges du tribunal correctionnel ou de la cour d'assises décident parfois de placer sous surveillance électronique mobile la personne majeure condamnée眘(97). Enfin, le tribunal de l'application des peines est compétent pour ordonner à titre de mesure de sûreté et aux seules fins de prévenir une récidive dont le risque paraît avéré, la surveillance judiciaire d'une personne dangereuse

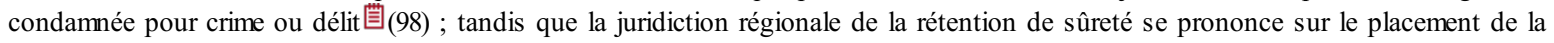


D'un point de vue passif, le critère personnel envisage, cette fois, la personne soumise à la surveillance. A priori, bon nombre de personnes sont susceptibles de faire l'objet d'une telle mesure. Leur statut importe peu d'ailleurs, le droit pénal prévoyant des surveillances à tous les stades de la procédure. Ainsi, des suspects peuvent être surveillés au stade des enquêtes de police, préliminaire ou de flagrance. Une fois l'action publique mise en mouvement et le juge d'instruction saisi, celui-ci pourra ordonner des interceptions téléphoniques 眘 (100), des sonorisations ou diverses mesures très intrusives participé comme auteurs ou complices à l'infraction pourront être surveillés par ce magistrat et par les officiers de police judiciaire agissant sur commission rogatoire. Menée à charge et à décharge, l'information a pour objectif de découvrir la vérité et de recueillir des preuves de l'infraction首(102). Il ne fait guère de doute que la surveillance, sous ses différents aspects, y occupe une place importante. Si elle concerne le suspect lato sensu, elle touche également le condamné. Dans certains cas, ce dernier est astreint à une surveillance lors de l'exécution de sa peine, voire une fois celle-ci purgée lorsqu'il présente des risques avérés de récidive. Les juges répressifs disposent, à cet égard, des différentes mesures précitées : surveillance judiciaire, surveillance de sûreté, etc. En somme, la surveillance ne se fonde pas sur un critère personnel clairement identifiable. Ce critère organique étant insuffisant à définir cette notion, il invite à en rechercher un autre.

Il apparaît alors concevable de se tourner vers un critère matériel, entendu comme celui qui se rattache aux faits commis. Lidée consiste à vérifier s'il existe un lien tangible entre la surveillance, d'une part, et l'infraction accomplie, d'autre part. Seules certaines infractions particulières permettent peut-être aux autorités publiques de surveiller les personnes et, accessoirement, les biens. On songe à la nature de l'infraction ou à ses modalités de commission, ce qui revient en fin de compte à regarder sa gravité. Le critère tiré de la gravité de l'infraction constituerait ainsi l'élément adéquat pour appréhender la surveillance. La loi n 2019-222 du 23 mars 2019 a justement harmonisé les régimes applicables à certains procédés intrusifs en imposant un seuil de gravité commun de l'infraction recherchée. Désormais, la géolocalisation et l'interception de correspondance émise par voie électronique sont applicables aux délits punis d'au moins trois ans d'emprisonnement. Ce seuil diminue pour la géolocalisation (cinq ans auparavant) et augmente pour les interceptions (deux ans auparavant), quels que soient le cadre et l'infraction concernés眘 (103). Mais à vrai dire, ce critère semble, lui-aussi, insuffisant. De très nombreuses infractions peuvent appeler l'exécution d'une surveillance. Pour s'en convaincre, il suffit d'examiner la liste des infractions visées à l'article 706-73 ou 706-73-1 du Code de procédure pénale菌(104).

Cette liste s'est même considérablement allongée depuis la loi n 2019-222 du 23 mars 2019 puisque celle-ci a permis l'assimilation des crimes, quels qu'ils soient, aux infractions commises en bande organisée visées par les deux textes précités. L'intitulé du titre XXV du livre IV du Code de procédure pénale s'applique désormais à la criminalité et à la délinquance organisées « et aux crimes ». «Une telle modification de l'intitulé permettra, dans de prochains textes, d'ajouter des crimes au sein de l'article 706-73, pour appliquer à l'enquête ou à l'instruction portant sur un crime commis par une personne seule, par exemple, les mesures dérogatoires prévues par ce titre XXV»苜(105). De toute façon, si les diverses infractions énumérées aux articles 706-73 et 706-73-1 du Code de procédure pénale s'avèrent graves, toutes n'ont pas le même degré de gravité, de sorte que leurs auteurs sont passibles de peines distinctes.

Il convient de surcroît de s'interroger sur le point commun entre les infractions à l'origine d'une surveillance policière et celles donnant lieu à une surveillance judiciaire. Variées à leur tour, ces dernières renferment en général de la violence ou une connotation sexuelle 菌(106). En résumé, la surveillance peut être diligentée pour une infraction grave, ce qui exclut en logique une simple contravention. Mais les infractions concernées sont tellement dissemblables que le critère tiré de leur gravité semble flou et aléatoire. Faut-il comprendre, sous cette qualification, uniquement les crimes et exclure les délits ? Le légis lateur retient une autre conception, ne serait-ce qu'à l'article 706-73-1 du Code de procédure pénale. À partir de quel seuil précis de pénalité encourue peut-on considérer l'infraction comme grave : cinq ou dix ans d'emprisonnement, quinze ans de réclusion criminelle ? L'interrogation ne peut assurément recevoir une réponse nette et tranchée. Loin d'être sûr et opérant, le critère matériel dévoile son insuffisance à l'instar du critère personnel. Il subsiste d'autres critères à explorer, même s'ils vont vite révéler des lacunes et leur incapacité à définir la notion de surveillance.

\section{B - L'insuffisance des critères temporel et formel}

Dans l'optique de découvrir un critère adapté à la surveillance, il est possible d'évoquer le critère temporel. Celui-ci doit être compris comme le moment auquel la surveillance intervient au cours du procès pénal. Si la mesure se déroule systématiquement à un moment déterminé et précis, il s'agit peut-être d'un élément à prendre en considération dans l'identification de son critère. Hormis les surveillances du domaine adminis tratif qui ne s'inscrivent pas dans le cadre d'une procédure pénale曾(107), la surveillance est susceptible d'avoir lieu pour confirmer la commission du délit et ante sententia. Dans cette situation, les actes d'enquête ou d'information comme les écoutes téléphoniques, la sonorisation ou la géolocalisation, servent à constater l'infraction, à réunir les preuves et à rechercher leurs auteurs. Toutes ces mesures constituent des surveillances à finalité probatoire. Celles appartenant à la seconde catégorie, destinées à prévenir le renouvellement de l'infraction, sont d'ordinaire décidées lors du prononcé de la peine, voire après celle-ci, en d'autres termes post sententia 首(108). La surveillance judiciaire et la surveillance de sûreté en témoignent parfaitement. Notion transversale du droit pénal, la surveillance apparait au cours des enquêtes de police judiciaire et de l'information, au moment de l'exécution de la peine et même une fois que le condamné l'a purgée. Il convient d'en conclure qu'elle ne repose pas sur un critère temporel précis. Ce dernier se montre en effet insuffisant à appréhender cette notion.

C'est la raison pour laquelle il s'avère tentant de recourir à un critère formel pour définir la surveillance. La forme de celle-ci peut se concevoir de deux manières distinctes. Dans une première acception, elle fait songer à la publicité ou non de la surveillance. Soit la mesure est clandestine, soit elle est apparente. Elle reste dissimulée afin de préserver l'efficacité des investigations, la doctrine qualifiant cette clandestinité « d'opératoire » dans la mesure où elle se rapporte aux procédures judiciaires首(109). Il en va ainsi au stade des enquêtes de police et de linstruction préparatoire眘(110). Une logique contraire gouverne celle prononcée lors du jugement pénal, durant l'exécution de la peine ou après que le condamné a expié celle-ci. Dans ce cas, il n'est plus question de récolter des éléments de preuve de linfraction, mais de surveiller la personne afin de lutter contre la récidive. La mesure devient apparente ou ostensible, comme l'atteste les surveillances judiciaire et de sûreté. Dès lors, le critère temporel de la surveillance possède une influence significative sur sa forme.

Dans une seconde acception, la forme de la surveillance renvoie à ses modalités d'exécution. La surveillance peut être traditionnelle et 
visuelle à l'instar des obligations auxquelles le condamné est astreint, des filatures et surveillances diverses ou des infiltrations 眘(111). Des constatations visuelles sont réalisées et des photographies souvent prises à cette occasion目(112). Il n'empêche que la plupart du temps la surveillance s'effectue à l'aide de la technologie : elle est de plus en plus menée grâce à des moyens techniques ou numériques placement sous surveillance électronique, la sonorisation, la géolocalisation, l'IMSI catcher ou la captation des données informatiques illustrent les moyens innovants dont disposent les autorités publiques pour surveiller les personnes et les biens. Somme toute, ces différentes formes de surveillance font ressortir le caractère coercitif des plus intrusives d'entre-elles ; tandis que d'autres, beaucoup moins attentatoires à la vie privée, s'avèrent non coercitives 首(114). En l'absence de critère formel clairement identifiable, la surveillance peine à masquer, une nouvelle fois, l'impossibilité de lui appliquer un critère adéquat. Aucun critère unique, ni même cumulé avec d'autres, n'apparaît satisfaisant et capable de la définir réellement.

$* * *$

Conforme à la réalité juridique, la surveillance est en définitive une notion fondamentale du droit pénal substantiel et formel. Son caractère transversal empêche toutefois de lui offrir une définition conceptuelle. Le seul véritable point commun entre les diverses surveillances consiste, au fond, en l'atteinte portée à la vie privée, ce qui est insuffisant pour définir cette notion. Celle-ci se révèle fuyante en raison de sa dualité de nature, mais également de l'absence d'un ou plusieurs critères opérants. Dès lors, la surveillance pourrait constituer une notion fonctionnelle à l'image de certains concepts du droit pénal眘(115). Son unité reste hélas introuvable sur ce terrain-là encore, puisqu'elle exerce une double fonction : établir la preuve de l'infraction d'un côté et prévenir la récidive de l'autre. Si une telle notion s'avère insaisissable, on assiste paradoxalement à une prolifération des surveillances. Leur développement constant et progressif apparait comme le résultat direct d'une double évolution amorcée en France depuis une quinzaine d'années. Il s'agit du dédoublement de la procédure pénale et de « l'affichage sans ambiguité d'une procédure pénale bis depuis la loi du 9 mars 2004 pour la criminalité organisée »; ainsi que $\mathrm{du}$ « dédoublement du droit pénal dans ses fondements [...] avec la consécration de la dangerosité comme justification de mesures qui échappent au droit des peines à la suite de la commission de certaines infractions dont la liste s'accroît au fil des réformes "目(116). A défaut de pouvoir fournir une définition pleinement satisfaisante de la surveillance en droit pénal, il convient de veiller à ce que le législateur et la jurisprudence encadrent strictement cette mesure intrusive et, au-delà, empêchent toute atteinte injustifiée aux libertés individuelles.

\author{
Mots clés : \\ PROCEDURE PENALE* Surveillance * Surveillance policière * Surveillance judiciaire * Surveillance de sûreté
}

(1) V. encore récemment, CEDH 13 sept. 2018, Big Brother Watch et a. c/ Royaume-Uni , n 58170/13, 62322/14 et 24960/15 (en anglais), D. 2018. 1916, obs. S. Lavric 首 ; ibid. 2019. 151, obs. J.-F. Renucci眘 ; ibid. 1673, obs. W. Maxwell et C. Zolynski首 ; AJ pénal 2018. 529, obs. A. Taleb-Karlsson眘, estimant que le système britannique d'interception massive et d'obtention de données de communications méconnait, par certains aspects, l'art. 8 de la Conv. EDH.

(2) Ce qui fait débat, y compris devant le juge européen : v. CEDH, gr. ch., 5 sept. 2017, nº 61496/08, Barbulescu c/ Roumanie, not. §120 s., AJDA 2017. 1639首 ; ibid. 2018. 150, chron. L. Burgorgue-Larsen首; D. 2017. 1709, et les obs.首 ; ibid. 2018. 138, obs. J.-F. Renucci苜; ibid. 1033, obs. B. Fauvarque-Cosson et W. Maxwell眘; ibid. 2019. 157, obs. J.-D. Bretzner et A. Aynès 首 ; JA 2017, nº 568, p. 40, étude J. Marfisi眘 ; Dr. soc. 2018. 455, étude B. Dabosville眘; Dalloz IP/IT 2017. 548, obs. E. Derieux眘; CEDH 22 févr. 2018, Libert c/ France, n $^{\circ}$ $588 / 13$, spéc. $\$ 47$ s., D. 2018. 1291, et les obs.眘, note J.-P. Marguénaud et J. Mouly 眘 ; ibid. 2019. 157, obs. J.-D. Bretzner et A. Aynès 眘 ; JA 2018, n 577, p. 42, étude D. Castel眘 ; Dr. soc. 2018. 455, étude B. Dabosville首 ; Dalloz IP/IT 2018. 511, obs. G. Péronne et E. Daoud眘.

(3) L. n 2015-912 du 24 juill. 2015, JO du 26 juill. 2015, p. 12735 ; R. Parizot, Surveiller et prévenir... à quel prix ? Loi n $2015-912$ du 24 juillet 2015 relative au renseignement, JCP 2015. 1077.

(4) V. entre autres, art. L. 228-1 s., L. 853-1 s., L. 854-1 s., R. 228-1 s. du CSI.

(5) V. C. pr. pén., art. 723-29 s.; C. pr. pén., art. 706-53-13 s.; C. pén., art. 131-36-9 s. du C. pr. pén. et 763-10 s.

(6) V. C. pr. pén., art. 706-80, 706-80-1 et 706-80-2, ces deux derniers textes ayant été créés par la L. n 2019-222 du 23 mars 2019 de programmation 2018-2022 et de réforme pour la justice, JO du 24 mars 2019, texte $\mathrm{n}^{\circ} 2$; J.-B. Perrier, Les (r)évolutions de la procédure pénale. À propos de la loi du 23 mars 2019 de programmation et de réforme pour la justice, D. 2019. $1061 \mathrm{~s}$. ; J. Pradel, Notre procédure pénale à la recherche d'une efficacité à toute vapeur -. Loi n ${ }^{\circ}$ 2019-222 du 23 mars 2019, JCP 2019. 406; E. Vergès, Réforme de la procédure pénale : une loi fleuve, pour une justice au gré des courants - À propos de la loi n ${ }^{\circ}$ 2019-222 du 23 mars 2019 de programmation 2018-2022 et de réforme pour la Justice, Dr. pénal 2019. Étude 12.

(7) V. par ex, art. 230-32 s., 706-81 s., 706-95 s. et 706-96 s. du C. pr. pén. Certains de ces textes sont supprimés et intégrés dans « les techniques spéciales d'enquête » (art. 46 et 109 de la L. n 2019-222 du 23 mars 2019, préc.).

(8) E. Verny, Des surveillances nouvelles en droit pénal, in Les droits et le Droit, Mél. dédiés à B. Bouloc, Dalloz, 2007, p. 1141 s., spéc. p. 1141. 
(9) V. art. L. 251-1 s. du CSI ; M.-N. Mornet, La vidéosurveillance et la preuve, PUAM, 2004, préf. F. Granet-Lambrechts.

(10) V. art. L. 621-1 du CSI ; C. Chassang, La preuve privée : un progrès en procédure pénale ?, in Les transformations de la preuve pénale, LGDJ, 2018, P. Beauvais et R. Parizot (dir.), p. 125 s.

(11) Elles peuvent même se contenter de surveiller les agissements d'un plaignant, le juge pénal pouvant en déduire leur absence de participation directe ou indirecte à l'obtention des enregistrements clandestins : v. Cass., ass. plén., 10 nov. 2017, n 17-82.028 (affaire du « chantage au roi du Maroc »).

(12) V. supra, notes $\mathrm{n}^{\circ} 4$ et 5 s'agissant des textes. Pour la jurisprudence, v. par ex, Crim. 9 mai 2018, $\mathrm{n}^{\circ}$ 17-866.38, Bull. crim. $\mathrm{n}^{\circ} 89$; Crim. 5 sept. 2018, $n^{\circ} 16-87.180$, Bull. crim. ${ }^{\circ} 148$.

(13) V. not., P. Conte, L'apparence en matière pénale, Thèse, Grenoble, 1984, 2 tomes, $\mathrm{n}^{\circ} 562$ s., p. 705 s., expliquant que la réalité juridique est en quelque sorte synonyme de droit positif et, formant un concept général, elle signifie la conformité au réel, à la vérité.

(14) L'étude concerne la seule notion de surveillance comme l'indique l'intitulé. Elle ne traite pas son régime juridique, même si certains de ses éléments devront être évoqués car ils seront utiles à la démonstration.

(15) Dictionnaire de français Larousse, v. surveillance.

(16) Vocabulaire juridique, Association H. Capitant, G. Cornu (dir), PUF, Quadrige, 12 éd., 2017, v. surveillance. V. aussi, A.-S. ChaventLeclère, La surveillance : une pratique encadrée ? Renseignement, écoute, vidéo-protection..., Procédures, 2016. Étude 1, spéc. $n^{\circ} 5$ et 6 , pour qui la surveillance est « un acte d'observation passif qui peut porter sur une personne ou un bien ».

(17) O. Desaulnay, R. Ollard, Le renseignement français n'est plus hors-la-loi. Commentaire de la L. n 2015-912 du 24 juill. 2015 relative au renseignement, Dr. pénal 2015. Étude 17, spéc. n 9.

(18) R. Parizot, art. préc., spéc. $n^{\circ} 5$ et les auteurs cités en note.

(19) Ibid.

(20) Cons. const. 23 juill. 2015, n 2015-713 DC, JO du 26 juill. 2015, p. 12751; JCP 2015. 981, étude M. Verpeaux, consid. 9 : « Le recueil de renseignement [...] par les services spécialisés de renseignement [...] relève de la seule police administrative ; qu'il ne peut donc avoir d'autre but que de préserver l'ordre public et de prévenir les infractions ; qu'il ne peut être mis en oeuvre pour constater des infractions à la loi pénale, en rassembler les preuves ou en rechercher les auteurs ».

(21) E. Verny, Du renseignement à la preuve pénale, in Les transformations de la preuve pénale, LGDJ, 2018, P. Beauvais et R. Parizot (dir.), p. 155 s., spéc. p. 155 et 156.

(22) R. Parizot, art. préc., nº 5 ; v. aussi, E. Vergès, La procédure pénale au temps des confluences, RSC 2018. 153 s., spéc. p. 155 苜 : « le renseignement peut fournir des indices de suspicions qui ouvrent la voie à la réalisation d'actes coercitifs ou intrusifs d'enquête ou d'instruction ». V. par ex., Crim. 9 nov. 2011, $\mathrm{n}^{\circ}$ 05-87.745 et n ${ }^{\circ}$ 09-86.381, Bull. crim. $\mathrm{n}^{\circ}$ 230, employant la notion de « renseignements » pour désigner des informations utiles à la réalisation d'actes d'enquête - des surveillances -, mais qui ne peuvent pas être utilisées à titre de preuve.

(23) Sur ce point : E. Vergès, Propos conclusifs. La preuve pénale transformée ?, in Les transformations de la preuve pénale, LGDJ, 2018, P. Beauvais et R. Parizot (dir.), p. 333 s., spéc. p. 336. Les surveillances, infiltrations, sonorisations, etc., « poursuivent une finalité probatoire, mais également préventive ».

(24) Sur ces notions : P. Collet, L'acte coercitifen procédure pénale, éd. Panthéon-Assas, coll. Thèses, 2018, préf. D. Rebut, $\mathrm{n}^{\circ} 142 \mathrm{~s}$. et $\mathrm{n}^{\circ}$ $189 \mathrm{~s}$

(25) G. Stefani, Lacte d'instruction, in Recueil d'études en hommage à L. Hugueney, Problèmes contemporains de procédure pénale, Sirey, 1964, p. 135 s., spéc. n 20, p. 153. 
(26) B. Bouloc, L'acte d'instruction, LGDJ, Bibliothèque de sciences criminelles, 1965, préf. G. Stefani, nº 467 et 468.

(27) B. Bouloc, op. cit., $\mathrm{n}^{\circ} 469$ et 470 .

(28) B. Bouloc, op. cit., $\mathrm{n}^{\circ} 473$.

(29) S. Guinchard, J. Buisson, Procédure pénale, LexisNexis, Manuel, $11^{\mathrm{e}}$ éd., 2018, $\mathrm{n}^{\circ}$ 2059. La pensée du professeur Bouloc semble avoir évolué, les actes d'instruction étant ceux « qui ont pour but la recherche et la réunion des preuves de l'infraction, qu'ils soient accomplis par les juridictions d'instruction ou même par des officiers de police judiciaire » (B. Bouloc, Procédure pénale, Dalloz, Précis, 26 éd., 2018, $n^{\circ} 242$ et $\left.n^{\circ} 524\right)$.

(30) C. pr. pén., art. 9-2, $2^{\circ}$ et $3^{\circ}$; v. par ex., Crim. 28 oct. 2014, $\mathrm{n}^{\circ} 13-86.413$, jugeant que la consultation du fichier national des permis de conduire constitue « un acte d'instruction ou de poursuite », interruptif de prescription de l'action publique.

(31) E. Verny, Des surveillances nouvelles en droit pénal, art. préc., p. 1142.

(32) V. CEDH 26 oct. 2006, Khoudobine c/ Russie, $\mathrm{n}^{\circ}$ 59696/00, §128.

(33) V. M.-N. Mornet, op. cit., $\mathrm{n}^{\circ} 100$.

(34) V. entre autres : C. pr. pén., art. 706-81, al. 2 (infiltration) ; Cass., ass. plén., 6 mars 2015, n 14-84.339, Bull. ass. plén., $n^{\circ} 2$ (sonorisation de cellules contiguës de garde à vue); A. Lepage, Les provocations sur Internet, in Les transformations de la justice pénale, Dalloz, coll. Thèmes et commentaires, 2014, S. Guinchard et J. Buisson (dir.), préf. V. Lamanda, p. 231 s., spéc. n 7 s., p. 236 s.

(35) V. H. Matsopoulou, Les nouveaux moyens de preuve au service de la criminalité organisée. À propos de la loi n $2016-731$ du 3 juin 2016, JCP 2016. 707, libres propos.

(36) M. Quéméner, Loyauté de la preuve et nouvelles technologies : entre exigences processuelles et efficacité répressive, in Les transformations de la justice pénale, Dalloz, Thèmes et commentaires, 2014, S. Guinchard et J. Buisson (dir.), préf. V. Lamanda, p. 247 s., spéc. p. 248. Sur l'insuffisance de l'art. 81 du C. pr. pén. comme fondement à des «mesures aussi intrusives »: M. Touillier, Légalité et preuve. Réflexions sur les vices et vertus de la légalisation des méthodes probatoires, in Les transformations de la preuve pénale, LGDJ, 2018, P. Beauvais et R. Parizot (dir.), p. 41 s., spéc. p. 45 et 46.

(37) CEDH 2 sept. 2010, n 35623/05, Uzun c/ Allemagne, §52 et 66, D. 2011. 724, obs. S. Lavric眘, note H. Matsopoulou眘 ; RSC 2011. 217, obs. D. Roets眘 ; H. Matsopoulou, La surveillance par géolocalisation à l'épreuve de la Conv. EDH, D. 2011. 724 s.; Adde, M.-N. Mornet, op. cit., $\mathrm{n}^{\circ} 98$, pour qui « la surveillance audiovisuelle rend possible la collecte d'une multitude de renseignements nominatifs lorsqu'elle comprend un dispositif d'enregistrement, c'est d'ailleurs un moyen de surveillance plus important que les écoutes téléphoniques ».

(38) Lors de la discussion du projet de loi relatif au renseignement, l'IMSI catcher a été défini comme « une antenne relais mobile factice qui se substitue, dans un périmètre donné, aux antennes relais des opérateurs permettant ainsi aux services (spécialisés de renseignement) de disposer des informations sur les terminaux qui y sont connectés » (Sénat, Rapp. $n^{\circ}$ 460, 20 mai 2015, P. Bas, p. 83). Quant à la captation de données à distance, elle permet aux enquêteurs d'utiliser, dans certains cas, des logiciels espions enregistrant les frappes au clavier ou des captures d'écran, à l'insu de l'utilisateur (M. Quéméner, Criminalité économique et financière. À l'ère numérique, Economica, Pratique du droit, 2015, préf. Y. Charpenel, $n^{\circ} 568$ s.).

(39) Les auteurs sont unanimes sur ce point : v. H. Matsopoulou, art. préc. ; M. Quéméner, op. cit., $n^{\circ} 526$ s. ; S. Sontag Koenig, Technologies de linformation et de la communication et défense pénale, Mare \& martin, Bibliothèque des thèses, 2015, préf. L. Cadiet, $\mathrm{n}^{\circ}$ 220, 236 et 283 ; R. Allain et alii, La preuve numérique, in Les transformations de la preuve pénale, LGDJ, 2018, P. Beauvais et R. Parizot (dir.), p. $141 \mathrm{~s}$.

(40) Art. prél., III, al. 6, ajouté par l'art. 44 de la loi n 2019-222 du 23 mars 2019, préc. Ces dispositions sont entrées en vigueur le $1^{\mathrm{er}}$ juin 2019. 
(41) V. par ex, Crim. 6 janv. 2015, Bull. crim. $n^{\circ} 5$ (sonorisation d'un domicile) ; Crim. 15 juin 2016, Bull. crim. $n^{\circ} 186$ (interceptions téléphoniques); Crim. 20 juin 2018, n 17-86.657, Bull. crim. nº 116 (géolocalisation et perquisition).

(42) V. par ex, en matière de sonorisation et de fixation d'images : Crim. 20 mars 2019, n 17-86.319 et n 18-87.250, à paraître au Bull. crim.

(43) V. not., C. pr. pén., art. 100 et 230-33 avant comme depuis la loi du 23 mars 2019, préc. ; v. aussi, J.-C. Saint-Pau, Les investigations numériques et le droit au respect de la vie privée, AJ pénal 2017.321眘 s.

(44) E. Verny, Des surveillances nouvelles en droit pénal, art. préc., p. $1146 \mathrm{~s}$.

(45) C. pr. pén., art. 142-5.

(46) R. Perrot, La notion d'acte juridictionnel : le pacte civil de solidarité et le rôle du greffier, RTD civ. 2003. 546眘.

(47) Sur lesquels il n'est cependant pas utile de revenir, ce débat classique étant connu. Pour un exposé des controverses : P. Collet, op. cit., $\mathrm{n}^{\circ} 190$ à 194 .

(48) V. J. Jugault, De la liaison du contentieux. Essai sur la genèse de l'acte juridictionnel, Thèse, Rennes, 1969, p. 10; G. Cornu, J. Foyer, Procédure civile, PUF, Thémis droit privé, $3^{\mathrm{e}}$ éd., 1996, p. 99 ; M. Bandrac, De l'acte juridictionnel, et de ceux des actes du juge qui ne le sont pas, in Le juge entre deux millénaires, Mél. P. Drai, Dalloz, p. 171 s., spéc. $\mathrm{n}^{\circ} 1$ et 5, p. 171 et 176; L. Cadiet, Rapport de synthèse, in Procédure civile et procédure pénale. Unité et diversité ?, Bruylant, 2014, S. Amrani-Mekki (dir.), p. 207 s., spéc. p. 220.

(49) G. Cornu, J. Foyer, op. et loc. cit.

(50) V. CEDH 26 juin 1986, Van Marle et a. c/ Pays-Bas, n 8543/79, 8674/79, 8675/79 et 8685/79, refusant d'appliquer ce texte en l'absence de contestation. Celle-ci doit du reste présenter certains caractères. Elle doit être « réelle et sérieuse ; elle peut concerner aussi bien l'existence même d'un droit que son étendue ou ses modalités d'exercice; enfin, l'issue de la procédure doit être directement déterminante pour un tel droit » (v. par ex., CEDH 25 nov. 1993, Zander c/ Suède, n 14282/88, AJDA 1994. 16, chron. J.-F. Flauss 眘.

(51) R. Kovar, La notion de juridiction en droit européen, in Gouverner, administrer, juger, Liber amicorum J. Waline, Dalloz, 2002, p. 607 s., spéc. $n^{\circ} 45$, p. 626.

(52) V. not., CEDH 22 oct. 1984, Sramek c/ Autriche, n 8790/79.

(53) V. D. D'ambra, L'objet de la fonction juridictionnelle : dire le droit et trancher les litiges, LGDJ, 1994, Bibliothèque de droit privé, t. 236, préf. G. Wiederkehr.

(54) J. Moury, De quelques aspects de l'évolution de la jurisdictio (en droit judiciaire privé), in Nouveaux juges, nouveaux pouvoirs, Mél. R. Perrot, Dalloz, 1996, p. 299 s. ; G. Wiederkehr, Qu'est-ce qu'un juge ?, in Nouveaux juges, nouveaux pouvoirs, Mél. R. Perrot, Dalloz, 1996, p. 575 s., spéc. p. 581 et 582.

(55) En ce sens : G. Wiederkehr, art. préc., p. 582 ; S. Rials, La fonction de juger. Ouverture : l'office du juge, Droits, nº 9, 1989, p. 3 s., spéc. p. 8 et 9 .

(56) X. Pin, La notion de juridiction (Le point de vue d'un pénaliste), in Procédure civile et procédure pénale. Unité et diversité ?, Bruylant, 2014, S. Amrani-Mekki (dir.), p. 57 s., spéc. p. 58 et 59.

(57) V. L. Cadiet, art. préc., p. 214 et 215, ajoutant : « [...] comme s'il n'y avait nul besoin de s'interroger sur ce qu'est une juridiction, sur ce qu'est un acte juridictionnel, à la différence de ce qui se passe en procédure civile ou en procédure administrative contentieuse ».

(58) L. Cadiet, art. préc., p. 221 et 224, pour qui « administrer la justice, c'est tout à la fois administrer les institutions et administrer les procédures, ce que mettent d'ailleurs en oeuvre les actes formant la catégorie composite des mesures d'administration judiciaire ». 
(59) V. R. Gassin, Les mesures d'administration judiciaire en procédure pénale, in Justices et droit du procès. Du légalisme procédural à l'humanisme processuel, Mél. S. Guinchard, Dalloz, 2010, p. 951 s., spéc. nº 14 s., p. 957 s.

(60) C. pén., art. 132-40.

(61) Le sursis avec mise à l'épreuve devient le « sursis probatoire » selon l'art. 80 de la L. n 2019-222 du 23 mars 2019 préc., mais conformément à l'article 109 de ladite loi, ces dispositions entrent en vigueur un an après la publication de la loi, soit le 24 mars 2020.

(62) Il faut se rappeler qu'« il y a dans tout jugement pénal, une réponse à la fois à un acte et à un homme. Cest là toute la particularité et la complexité de la justice pénale. Le juge pénal exerce toujours son office dans une double dimension collective (en réprouvant la transgression d'un interdit) et individuelle (en infligeant une sanction à un auteur selon sa personnalité), en se prononçant à la fois sur le passé (la culpabilité) et sur l'avenir (la dangerosité). Un jugement pénal est donc toujours traversé par une logique à la fois rétrospective et prospective, qui lui donne un côté sacré et qui explique que la justice pénale ait toujours été plus ritualisée que la justice civile (...)» (X. Pin, art. préc., p. 62, qui souligne).

(63) Suite à la L. n 2019-222 du 23 mars 2019, préc., l'art. 132-26-1 va disparaître le 24 mars 2020. Le C. pén. sera modifié et comportera une sous-section 1, intitulée « De la détention à domicile sous surveillance électronique, de la semi-liberté et du placement à l'extérieur » (art. 132-25 s. du C. pén.).

(64) Texte modifié par la L. n 2019-222 du 23 mars 2019, préc.

(65) L'al. 2 de ce texte prévoit que « le juge de l'application des peines peut subordonner la libération conditionnelle du condamné à l'exécution, à titre probatoire, d'une mesure de placement sous surveillance électronique, pour une durée n'excédant pas un an. La mesure de placement sous surveillance électronique peut être exécutée un an avant la fin du temps d'épreuve prévu à l'article 729 ou un an avant la date à laquelle est possible la libération conditionnelle prévue à l'article 729-3».

(66) Ce suivi, qualifié de « peine pas comme les autres » par Couvrat, a pour principal objet la surveillance des délinquants sexuels. V. J. Alix, Une liaison dangereuse. Dangerosité et droit pénal en France, in La dangerosité saisie par le droit pénal, PUF et IRJS Éditions, 2011, G. Giudicelli-Delage et C. Lazerges (dir.), p. 49 s., spéc. p. 60.

(67) V. Cons. const. 8 déc. 2005, n² 2005-527 DC ; D. 2006. 966, note F. Rouvillois.

(68) C. pén., art. 131-36-9 et 131-36-10 ; Y. Mayaud, Droit pénal général, PUF, Droit fondamental, 6 éd., 2018, n 564.

(69) C. pén., art. 131-36-12, al. 1.

(70) C. pén., art. 131-36-12, al. 2.

(71) Sur la différence « technique » entre un placement sous surveillance dit « fixe » et un placement sous surveillance électronique « mobile » (PSEM) : S. Sontag Koenig, op. cit., nº 271 s., spéc. n² 272 et 273 : « À la différence du PSE, le PSEM combine l'utilisation de deux technologies. S'il fait appel au réseau téléphonique satellitaire, il est couplé avec le GPS, permettant, à l'inverse du PSE de connaître à tout moment le lieu où se trouve la personne porteuse du bracelet ».

(72) Y. Mayaud, op. et loc. cit., le soulignant. Il n'en demeure pas moins que l'on est en présence, dans les deux cas, d'actes juridictionnels. Pour une définition de ce concept : L. Grégoire, Les mesures de sûreté. Essai sur l'autonomie d'une notion, Institut universitaire Varenne, coll. des Thèses, 2015, préf. P. Bonfils, $\mathrm{n}^{\circ} 25:$ : Les mesures de sûreté peuvent [...] être définies comme des sanctions pénales déterminées par la loi pénale, prononcées par une juridiction pénale en raison de la dangerosité de l'individu et ordonnées dans le dessein exclusif de prévenir la commission d'une infraction pénale que la dangerosité rend probable».

(73) V. H. Matsopoulou, Le renouveau des mesures de sûreté, D. 2007. 1607 s. ; C. Lazerges, Lirruption de la dangerosité dans les décisions du Conseil constitutionnel, in La dangerosité saisie par le droit pénal, PUF et IRJS Éditions, 2011, G. Giudicelli-Delage et C. Lazerges (dir.), p. 79 s., not. p. 80. Sur la notion de dangerosité : J. Alix, O. Cahn, La fin de la preuve ? La preuve dénaturée à l'épreuve du concept de dangerosité, in Les transformations de la preuve pénale, LGDJ, 2018, P. Beauvais et R. Parizot (dir.), p. 301 s., not. p. 303 à 306.

(74) J. Alix, art. préc. 
(75) G. Giudicelli-Delage, Conclusion. Un monde (simplement) habitable..., in La dangerosité saisie par le droit pénal, PUF et IRJS Éditions, 2011, G. Giudicelli-Delage et C. Lazerges (dir.), p. 281 s., not. p. 295 : « Les mesures pénales de neutralisation - [...] ou d'"incapacitation" - se présentent majoritairement comme des mesures personnelles s'articulant autour d'un axe "Eloigner/Surveiller/Détenir" ».

(76) On prendra soin de la distinguer de la surveillance judiciaire, peine propre aux personnes morales (C. pén, art. 131-39, al. 1, $3^{\circ}$ et $131-$ 46). Ces dernières peuvent, à titre de peine complémentaire criminelle ou correctionnelle, être condamnées pour cinq ans maximum à la surveillance judiciaire.

(77) L'étude ne gagnerait rien à rappeler, de manière exhaustive, le domaine et le régime de cette mesure. V. Y. Mayaud, op. cit., $\mathrm{n}^{\circ} 596 ; \mathrm{J}$. Leroy, Droit pénal général, LGDJ, Manuel, 7 éd., 2018, nº 845 et 846 ; O. Décima, S. Detraz, E. Verny, Droit pénal général, LGDJ, Cours, $3^{\mathrm{e}}$ éd., 2018, nº 620 et 757.

(78) Elle ne permet donc pas d'étendre le contrôle social au-delà de la durée de la peine prononcée (v. sur ce point, J. Alix, art. préc., p. 62 et 63).

(79) C. pr. pén., art. 723-30. D'après le Haut conseil, « la surveillance judiciaire, y compris lorsqu'elle comprend un placement sous surveillance électronique mobile [...] repose non sur la culpabilité du condamné, mais sur sa dangerosité ; qu'elle a pour seul but de prévenir la récidive ; qu'ainsi la surveillance judiciaire ne constitue ni une peine ni une sanction » (Cons. const. 8 déc. 2005, $n^{\circ} 2005-527$ $\mathrm{DC}$, préc.).

(80) C. pr. pén., art. 723-32. ; v. Crim. 11 mai 2017, n $16-84.383$, Bull. crim. nº 139.

(81) C. pr. pén., art. 723-32, al. 2. D'autres actes juridictionnels existent : le juge de l'application des peines peut justement modifier par ordonnance les obligations auxquelles le condamné est astreint, mettre fin à ces obligations, mais aussi, au cas où le condamné ne respecte pas la décision, retirer tout ou partie de la réduction de peine dont il a bénéficié et ordonner sa réincarcération.

(82) L'intéressé doit être informé par le président de la juridiction régionale de la rétention de sûreté que le PSEM ne peut être mis en oeuvre sans son consentement mais que, à défaut ou s'il manque à ses obligations, il pourra être placé dans un centre socio-médico-judiciaire (C. pr. pén., art. 706-53-19, al. 5). Sur l'exclusion de compétence des juridictions de l'application des peines au profit de juridictions spécialisées dans le prononcé de la surveillance ou rétention de sûreté : L. Gregoire, op. cit., ${ }^{\circ} 406$ s. et n 434.

(83) C. pr. pén., art. 723-37. La preuve, d'ordinaire inscrite dans un processus rétrospectif, suit une « démarche prospective » lorsque le juge doit évaluer la dangerosité d'une personne, les chances de réinsertion ou le risque de récidive. « Le mécanisme probatoire s'intègre dans un modèle de prédiction d'un comportement futur » (E. Vergès, Propos conclusifs [...] art. préc., p. 335). V. J. Alix, O. Cahn, art. préc., p. 305 à 308.

(84) C. pr. pén., art. 706-53-19, al. 1.

(85) C. pr. pén., art. 706-53-19, al. 3 et 4.

(86) Cons. const. 21 févr. 2008, n 2008-562 DC, Loi relative à la rétention de sûreté et à la déclaration d'irresponsabilité pénale pour cause de trouble mental, consid. 9 et 12 ; Y. Mayaud, La mesure de sûreté après la décision du Conseil constitutionnel n ${ }^{\circ} 2008-562$ DC du 21 févr. 2008, D. 2008. 1359 s. ; C. Lazerges, La rétention de sûreté : le malaise du Conseil constitutionnel, RSC 2008. 731 眘 s.

(87) Ibid. Le recours est porté devant la JNRS, composée de trois conseillers à la Cour de cassation. Sa décision est motivée et susceptible d'un pourvoi en cassation.

(88) V. aussi, L. Gregoire, op. cit., nº 215 et nº 257.

(89) Bien que des surveillances émanant d'autorités privées existent, comme les employeurs, mieux vaut se concentrer uniquement sur celles pratiquées par les autorités publiques, seules investies de véritables pouvoirs de contrainte et du droit d'attenter à la vie privée des individus.

(90) Les possibilités d'action, ou même d'inaction volontaire, sont accrues depuis la L. n 2019-222 du 23 mars 2019 ayant créé les art. 706- 
(92) V. C. pr. pén., art. 230-32 in fine : « La géolocalisation est mise en place par l'officier de police judiciaire ou, sous sa responsabilité, par l'agent de police judiciaire, ou prescrite sur réquisitions de l'officier de police judiciaire [...] ». Sur la nécessité de justifier le recours à la procédure d'urgence : v. Crim. 20 juin 2018, $\mathrm{n}^{\circ}$ 17-86.657, Bull. crim. $\mathrm{n}^{\circ} 116$.

(93) C. pr. pén., art. 706-95-11 s., créés par la L. n 2019-222 du 23 mars 2019, spéc. l'art. 706-95-17 : « Les techniques spéciales d'enquête mentionnées à la présente section sont mises en place par l'officier de police judiciaire commis par le juge d'instruction ou requis par le procureur de la République ou, sous sa responsabilité, par l'agent de police judiciaire ».

(94) V. not., C. pr. pén., art. 706-98.

(95) Le juge constitutionnel a amputé le projet de loi de mars 2019 de dispositions qui lui sont apparues disproportionnées. Il a notamment refusé l'extension aux crimes des techniques spéciales d'enquête, ces procédures « particulièrement intrusives » n'étant pas adaptées à ce type d'infractions « ne présentant pas nécessairement un caractère de particulière complexité » : Cons. const. 21 mars 2019, $\mathrm{n}^{\circ} 2019-778$ DC, Loi de programmation 2018-2022 et de réforme pour la justice, JO du 24 mars 2019, texte $n^{\circ} 4, \S 161$ à 166 . Sur ce point, E. Vergè, Réforme de la procédure pénale [...], art. préc., $\mathrm{n}^{\circ} 13 ; \mathrm{J}$. Pradel, Notre procédure pénale à la recherche [...], art. préc., $\mathrm{n}^{\circ} 24$.

(96) C. pr. pén., art. 723-7, modifié par la L. n 2019-222 du 23 mars 2019.

(97) C. pén., art. 131-36-9 s.

(98) C. pr. pén., art. 723-29s.

(99) C. pr. pén., art. 706-53-19. Pour des développements sur ces diverses mesures, v. supra.

(100) V. Crim. 20 juin 2018, préc.

(101) Le législateur de 2019 a souhaité étendre le domaine des interceptions de correspondances émises par la voie des communications électroniques en permettant d'y procéder lors d'une enquête, préliminaire ou de flagrance, pour toute infraction dès lors qu'elle est punie d'au moins trois ans d'emprisonnement, seuil retenu pour les interceptions effectuées au cours d'une instruction. Cependant, le Cons. const. a exercé sa censure à ce sujet. Ces interceptions sont uniquement possibles au cours d'une information ou bien d'une enquête mais portant sur des faits relevant de la criminalité ou de la délinquance organisées. V. Cons. const. 21 mars 2019, n² 2019-778 DC, préc., § 142 à 147.

(102) C. pr. pén., art. 81 ; v. Crim. 11 déc. 2018, n 18-82.365, Bull. crim. n 211 ; JCP 2019. 496, n 12, obs. J.-B. Perrier, jugeant que le juge d'instruction tire de l'art. 81 du C. pr. pén., interprété à la lumière de l'art. 8 de la Conv. EDH, « le pouvoir de faire procéder à une vidéosurveillance sur la voie publique aux fins de rechercher des preuves des infractions dont il est saisi, à l'encontre des personnes soupçonnées de les avoir commises, une telle ingérence dans la vie privée présentant, par sa nature même, un caractère limité et étant proportionnée au regard de l'objectif poursuivi (...)».

(103) C. pr. pén., art. 230-32, modifié et C. douanes, art. 67 bis-2, modifié (géolocalisation) ; C. pr. pén., art. 100. Sur la question, E. Vergès, art. préc., $\mathrm{n}^{\circ} 15 ; \mathrm{J}$. Pradel, art. préc., $\mathrm{n}^{\circ} 17$.

(104) Il s'agit, parmi d'autres, du crime de meurtre commis en bande organisée, des crimes et délits de trafic de stupéfiants, de ceux constituant des actes de terrorisme, du crime de détournement d'aéronef, de navire ou de tout autre moyen de transport commis en bande organisée, etc.

(105) J.-B. Perrier, Les (r)évolutions de la procédure pénale [...], art. préc., spéc. $\mathrm{n}^{\circ} 6$, ajoutant que « sans attendre ces hypothèses qui pourront être textuellement introduites, la loi du 23 mars 2019 étend à tout crime l'accès à distance aux correspondances stockées (C. pr. pén., art. 706-95-1 et 706-95-2) ». En revanche, une telle extension a été refusée par le juge constitutionnel s'agissant des techniques spéciales d'enquête (v. supra, note $\mathrm{n}^{\circ} 94$ ). 
(106) Ainsi, la peine complémentaire de suivi socio-judiciaire a été créée par la L. du 17 juin 1998 après différentes affaires de pédophilie et des meurtres assortis de viols sur des enfants, commis par des récidivistes (C. pén., art. 131-36-1 s.). Elle oblige le condamné à se soumettre, sous le contrôle du juge de l'application des peines et pendant une durée déterminée par la juridiction de jugement, à des mesures de surveillance et d'assistance en vue de prévenir la récidive. Depuis 2005, cette peine peut comprendre, à titre de mesure de sûreté, le placement sous surveillance électronique mobile.

(107) Sur lesquelles, v. supra, I, A.

(108) V. supra, le plan de la $1^{\text {re }}$ partie sur cette dichotomie.

(109) D. Dechenaud, La clandestinité en procédure pénale, Mél. en l'honneur du professeur J.-H. Robert, LexisNexis, 2012, p. 213 s., spéc. $\mathrm{n}^{\circ} 6$ et n ${ }^{\circ} 17$ s. ; J.-C. Saint-Pau, art. préc.

(110) V. néanmoins, D. Dechenaud, art. préc., $\mathrm{n}^{\circ} 18$, faisant observer que « la clandestinité n'est donc pas la règle lors de la phase préparatoire du procès, non seulement parce que le principe du contradictoire s'y oppose, mais aussi parce que la publicité y progresse. Cependant, la recherche de l'efficacité peut conduire à déroger à l'exigence d'un débat contradictoire. Il arrive que l'autorité judiciaire avance masquée, afin de parvenir à la découverte de la vérité».

(111) C. pr. pén., art. 706-80 s. ; Adde, C. pr. pén., art. 694-18, $3^{\circ}$, mentionnant l'art. 40 de la Convention d'application des accords de Schengen du 19 juin 1990 prévoyant l'observation transfrontalière.

(112) V. Crim. 23 janv. 2013, $\mathrm{n}^{\circ}$ 12-85.059, Bull. crim. $\mathrm{n}^{\circ} 29$ (constatations visuelles); Crim. 21 mars 2007, $\mathrm{n}^{\circ}$ 06-89.444, Bull. crim. $\mathrm{n}^{\circ} 89$ (photographies clandestines, au moyen d'un téléobjectif, de plaques d'immatriculation de véhicules aux fins d'identification des titulaires des cartes grises).

(113) Certains auteurs opèrent cette distinction en évoquant « la surveillance physique » et « la surveillance à distance ». V. A.-S. Chavent-Leclère, La surveillance : une pratique encadrée ? (...), art. préc., $\mathrm{n}^{\circ} 41 \mathrm{~s}$.

(114) Rappr., M. Quéméner, Criminalité économique et financière, À l'ère numérique, op. cit., n 532, notant que « même en l'absence de loi, la mission de la police judiciaire issue de l'article $14 \mathrm{du}$ Code de procédure pénale lui permet de procéder à des surveillances sans coercition ni contrainte "; J. Alix, Une liaison dangereuse (...), art. préc., p. 73 : " Quant aux diverses formes de surveillance, elles sont certes restrictives de liberté, mais la restriction est parfois extrêmement lourde, notamment lorsque la surveillance s'effectue par le biais d'un bracelet électronique ».

(115) Par ex, les décisions provisoires. V. E. Bonis-Garçon, Les décisions provisoires en procédure pénale, PUAM, 2002, préf. P. Conte.

(116) C. Lazerges, L'irruption de la dangerosité (...), art. préc., p. 87. 